www.elsevier.com/locate/ces

\title{
Theoretical analysis on diffusional release from ellipsoidal drug delivery devices
}

\author{
Bo-Tau Liu ${ }^{\mathrm{a}, *}$, Jyh-Ping Hsu ${ }^{\mathrm{b}}$ \\ a Daxon Technology Inc., Taoyuan, Taiwan 33341 \\ ${ }^{\mathrm{b}}$ Department of Chemical Engineering, National Taiwan University, Taipei, Taiwan 10617
}

Received 1 June 2005; received in revised form 9 September 2005; accepted 10 October 2005

Available online 23 November 2005

\begin{abstract}
A theoretical analysis is conducted on the kinetics of diffusional release of an ellipsoidal drug delivery device. By choosing appropriate coordinates, the present analysis is applicable to devices of various shapes such as prolates, oblates, disks, and rod-like shapes. We show that, regardless of the shape of a device, the cumulative fraction of drug release can be expressed in a concise, yet general form. The performance of a widely adopted empirical relation in the literature for the description of the temporal variation in the amount of drug released is compared with that of the present study. The result of numerical simulation reveals that, although the former is satisfactory for the case when the cumulative fractional drug release is below $60 \%$, it may lead to inappropriate description for the release kinetics considered in this study.
\end{abstract}

(C) 2005 Elsevier Ltd. All rights reserved.

Keywords: Diffusional release; Ellipsoidal device; Moving-boundary problem

\section{Introduction}

The kinetics of controlled release has been investigated extensively in the literature for both drug delivery and transport of bioactive agents. Often, the release processes involved in controlled release are assumed to be governed by Fickian diffusion, Case II transport, or non-Fickian diffusion (Crank, 1975). Among these mechanisms, the first one, which is based on Fick's law, has been considered most often thanks to its practical significance (Paul and McSpadden, 1976; Narasimhan and Langer, 1997; Lee, 1980; Abdekhodaie, 2002; Asrar et al., 2004; Zhou et al., 2004). However, due to mathematical difficulty involved, most of the relevant analyses are limited to simple geometries such as planar, cylindrical, and spherical devices (Higuchi, 1961; Paul and McSpadden, 1976; Lee, 1980;

\footnotetext{
* Corresponding author. Tel.: +8863374 8800x2125; fax: +88633748888.
}

E-mail address: btliu@ntu.edu.tw (B.-T. Liu).
Liu and $\mathrm{Xu}, 2004)$. The empirical expression below is usually adopted to describe release kinetics

$$
\frac{M_{t}}{M_{\infty}}=k t^{n}
$$

where $M_{t}$ and $M_{\infty}$ are, respectively, the amount of drug released at time $t$ and that as $t \rightarrow \infty, k$ is a kinetic constant, and the exponent $n$ is a shape-dependent parameter. Ritger and Peppas (1987a,b) pointed out that for Fickian diffusion, $n$ is $0.5,0.45$, and 0.43 for slabs, cylinders, and spheres, respectively, and Eq. (1) is suitable for $M_{t} / M_{\infty}<0.6$. These analyses are restricted to drug loading up to the solubility of drug. Beyond that limit, a moving-boundary or Stefan problem should be considered.

In this study, an attempt is made to extend the analysis on the diffusional release for devices of simple shapes to a more general ellipsoidal device subjected to a moving-boundary condition. That is, we consider a problem that is closer to practical pharmaceutical applications than previous ones. The applicability of Eq. (1) in the present device is also discussed. 


\section{Analysis}

In a diffusion-controlled drug delivery system the concentration of drug in the liquid phase, $C$, is governed by Fick's second law

$\frac{\partial C}{\partial t}=D \nabla^{2} C$

where $\nabla^{2}$ is the Laplace operator and $D$ is the diffusivity of drug. The boundary conditions associated with Eq. (2) are

$C=C_{S}$ at a solid-liquid interface,

$C=C_{b}$ in a bulk liquid phase,

where $C_{s}$ and $C_{b}$ are, respectively, the drug concentration at the solid-liquid interface and in the bulk liquid phase. Referring to Fig. 1, we consider an ellipsoid device described by the ellipsoidal coordinates $\{\xi, \eta, \Phi\}$, where $\xi, \eta$, and $\Phi$ are, respectively, the radial, the angular, and the rotational coordinates. Let $a$ be the distance between the focus and the center of the device. The domains for the ellipsoidal coordinates are (Flammer, 1957): $(1+j) / 2 \leqslant \xi<\infty,-1 \leqslant \eta \leqslant 1$, and $0 \leqslant \Phi \leqslant 2 \pi$, where $j=1$ for prolates and $j=-1$ for oblates. Let us consider the transformation (Ham, 1959; Liu and Hsu, 1995)

$$
\begin{aligned}
& x=a \sqrt{\left(\xi^{2}-j\right)\left(1-\eta^{2}\right)} \cos \Phi \sqrt{1-t / \tau}, \\
& y=a \sqrt{\left(\xi^{2}-j\right)\left(1-\eta^{2}\right)} \sin \Phi \sqrt{1-t / \tau}, \\
& z=a \xi \eta \sqrt{1-t / \tau} .
\end{aligned}
$$

In these expressions, $\{x, y, z\}$ is the Cartesian coordinates, and $\tau$ is a characteristic total release time at which the solid phase
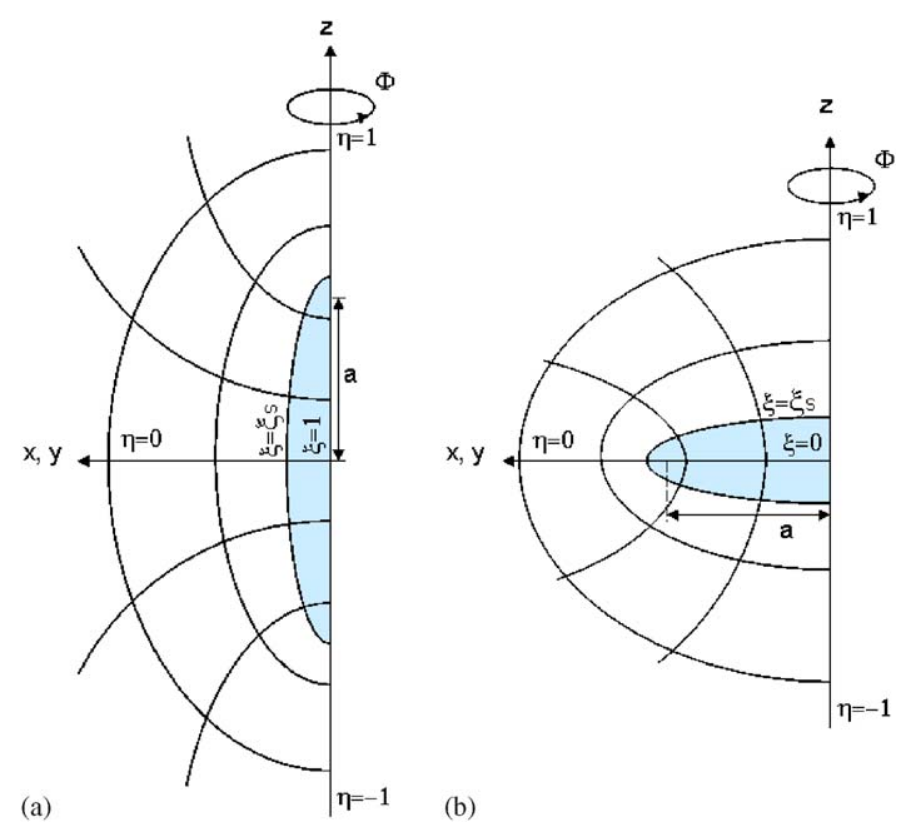

(a)

Fig. 1. Schematic representation of the ellipsoidal coordinates adopted for (a) prolates and (b) oblates. disappears, that is, the solid-liquid interface reaches the origin of the coordinates. Note that while $\{x, y, z\}$ forms a timedependent coordinate system, $\{\xi, \eta, \Phi\}$ is time-independent. Under conditions of practical significance, the amount of drug loaded is usually much greater than the solubility of drug, the time scale for the release process is long, and therefore, the rate of movement of the solid-liquid interface is relatively slow compared to that of drug diffusion. In this case, a pseudo-steady state can be assumed, the left-hand side of Eq. (2) vanishes, and the solution to the resultant equation, in ellipsoidal coordinates, is

$C=\left(C_{s}-C_{b}\right) \frac{f_{j}(\xi)}{f_{j}\left(\xi_{s}\right)}+C_{b}$,

where $\xi_{s}$ represents the position of the solid-liquid interface, and

$f_{j}(\xi)= \begin{cases}\frac{1}{2} \operatorname{Ln} \frac{\xi-1}{\xi+1}, & j=1, \\ \tan ^{-1} \xi-\frac{\pi}{2}, & j=-1 .\end{cases}$

The temporal variation in the position of the solid-liquid interface is described by the mass balance equation

$\left(C_{0}-C_{S}\right)\left(\mathbf{v} \cdot \mathbf{e}_{1}\right)=D \nabla C \cdot \mathbf{e}_{1}$

Here, $C_{0}$ is the concentration of drug loaded, $\mathbf{v}$ is the velocity vector associated with the movement of the solid-liquid interface, $\nabla$ is the gradient operator, and $\mathbf{e}_{i}$ is the covariant base vector, $i=1,2$, and 3 for $\xi, \eta$, and $\Phi$ axes, respectively. Based on Eqs. (3)-(5) and the tensor relation

$\mathbf{e}^{m} \cdot \mathbf{e}_{i}= \begin{cases}1 & \text { if } m=i, \\ 0 & \text { if } m \neq i\end{cases}$

Where $\mathbf{e}^{m}$ is the contravariant base vector, $m=1,2$, and 3 for $\xi, \eta$, and $\Phi$ axes, respectively. We obtain

$\mathbf{v} \cdot \mathbf{e}=-\frac{a^{2}}{2 \tau} \xi_{s}(1-t / \tau)^{-1 / 2}$,

Substituting Eqs. (6) and (10) into Eq. (8) yields

$\tau=-\frac{a^{2}}{2 D} \rho \xi_{s} f_{j}\left(\xi_{s}\right)\left(\xi_{s}^{2}-j\right)$,

where $\rho=\left(c_{0}-c_{s}\right) /\left(c_{s}-c_{b}\right)$. According to this expression, the value of $\tau$ depends upon the shape of a device, the diffusivity of drug, and the ratio $\rho=$ (overloaded drug concentration/maximum concentration difference for diffusion). Note that $\tau$ is proportional to the square of the linear size of a device, and since the time scale is eliminated from Eq. (8), the transformation expressed in Eqs. (3)-(5) simplifies a moving-boundary problem to a fixed-boundary one, which is much easier to solve. This transformation implies that the present one-dimensional, temporal variation in the position of the solid-liquid interface is proportional to $(1-t / \tau)^{1 / 2}$. According to the 
transformation expressed in Eqs. (3)-(5), the metric coefficients for the present ellipsoidal coordinates system can be written as

$g_{\xi \xi}=a^{2}\left(\frac{\xi^{2}-j \eta^{2}}{\xi^{2}-j}\right)\left(1-\frac{1}{\tau}\right)$

$g_{\eta \eta}=a^{2}\left(\frac{\xi^{2}-j \eta^{2}}{1-\eta^{2}}\right)\left(1-\frac{1}{\tau}\right)$

$g_{\Phi \Phi}=a^{2}\left(\xi^{2}-j\right)\left(1-\eta^{2}\right)\left(1-\frac{t}{\tau}\right)$.

If we let $M_{t}$ be the total amount of drug contained in a device subtracts the residual amount of drug in the device, then the amount of drug released at time $t$ can be evaluated by

$$
\begin{aligned}
M_{t}= & a^{3} \int_{0}^{2 \pi} \int_{-1}^{1} \int_{0}^{\xi_{s}}\left(C_{0}-C_{b}\right)\left(\xi^{2}-j \eta^{2}\right) \mathrm{d} \xi \mathrm{d} \eta \mathrm{d} \Phi \\
& -a^{3} \int_{0}^{2 \pi} \int_{-1}^{1} \int_{0}^{\xi_{s}}\left(C_{0}-C_{b}\right)\left(\xi^{2}-j \eta^{2}\right) \\
& \times(1-t / \tau)^{3 / 2} \mathrm{~d} \xi \mathrm{d} \eta \mathrm{d} \Phi \\
= & \frac{4 \pi}{3}\left(C_{0}-C_{b}\right) a^{3} \xi_{s}\left(\xi_{s}^{2}-j\right)\left(1-(1-t / \tau)^{3 / 2}\right) .
\end{aligned}
$$

The cumulative fractional release of drug, $M_{t} / M_{\infty}$, is

$\frac{M_{t}}{M_{\infty}}=1-\left(1-\frac{t}{\tau}\right)^{3 / 2}$

This expression is similar to that for Case II transport (Hopfenberg, 1976; Ritger and Peppas, 1987b; Costa and Lobo, 2001), where the exponent is 1,2 , and 3 for slabs, cylinders, and spheres. Eq. (16) suggests that the cumulative fractional release of drug can be approximated by a general form, which is independent of the shape of a device. The influences of the physical properties of the present problem such as the diffusivity of drug, the initial amount of drug loaded, and the shape of a device can all be lumped into the characteristic total release time $\tau$.

\section{Results and discussion}

Although the present analysis is based on an ellipsoidal device, the results for devices of other shapes which might be of practical significance can be deduced from the present result through adjusting the value of $\xi_{s}$. For example, a spherical device can be recovered from the present one by assuming a large $\xi_{s}$. On the other hand, if $\xi_{s}$ is small, we have a rod-like device if $j=1$, and a disk-shaped device if $j=-1$. As mentioned previously, even the cases for the various geometry of a device, the corresponding release kinetics can always be reduced to the close forms by Eqs. (11) and (16).

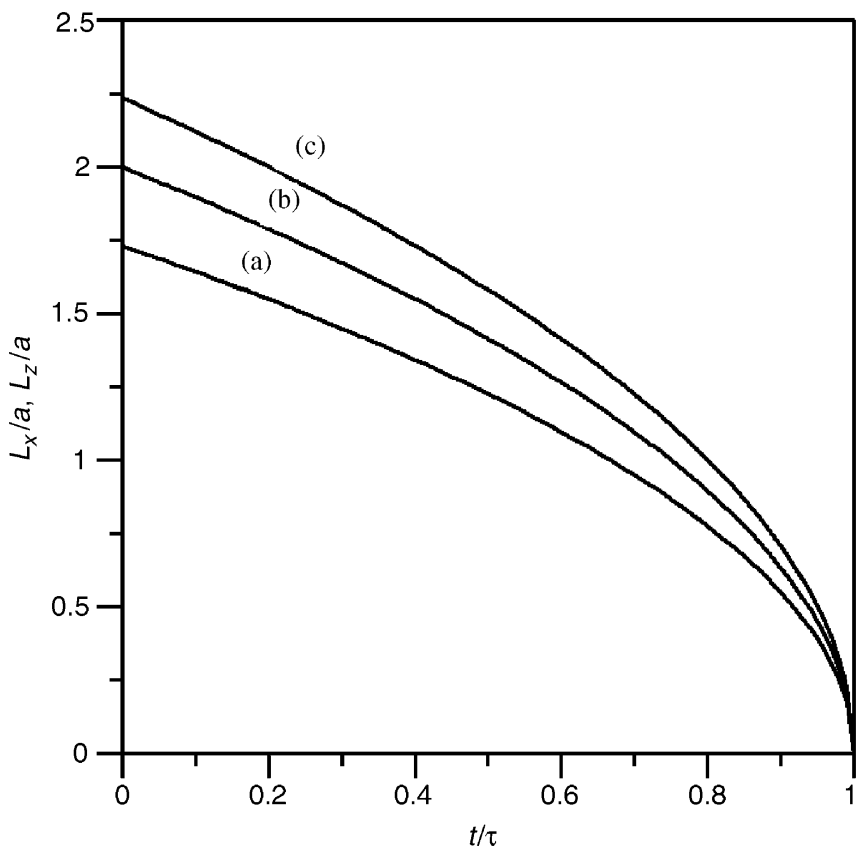

Fig. 2. Temporal variations in the sizes of a device for the case when $\xi_{s}=2$. (a) $L_{x}$ for a prolate device, (b) $L_{z}$ for a prolate or an oblate device, (c) $L_{x}$ for an oblate device.

According to Eqs. (3)-(5), the linear sizes of a device are

$L_{x}=L_{y}=a \sqrt{\left(\xi_{s}^{2}-j\right)(1-t / \tau)}$,

$L_{z}=a \xi_{s} \sqrt{1-t / \tau}$.

Based on these expressions, the values of $a$ and $\xi_{s}$ can be evaluated from given initial drug-loaded conditions. Fig. 2 shows the simulated temporal variations of $L_{x}$ and $L_{z}$ for both a prolate and an oblate device. This figure indicates that the longer the release time the faster the linear rate of release. Also, the result of $L_{x}$ and $L_{z}$ show that the larger the curvature, the faster the linear rate of release. The latter is because the release of drug in the present problem is diffusion controlled, and therefore, the larger the curvature the greater the driving force.

Eq. (1) is widely used to describe the release kinetics of drug delivery, especially when the exact release mechanism is unknown. Let us define the root-mean-square deviation of the result based on Eq. (1) from the corresponding exact result based on Eq. (16), $\langle\varepsilon\rangle$, as

$\langle\varepsilon\rangle=\sqrt{\frac{\int_{0}^{T_{0.6}}\left(1-(1-T)^{3 / 2}-k_{1} T^{n}\right)^{2} \mathrm{~d} T}{T_{0.6}}}$,

where $T=t / \tau, k_{1}=k \tau^{n}$, and $T_{0.6}=0.4571 . T_{0.6}$ denotes the value of $T$ at which the amount of release reaches $60 \%$. of the total amount of release, that is, $M_{t} / M_{\infty}=0.6$. The optimum value of $k_{1}, \hat{k}_{1}$, at which $\langle\varepsilon\rangle$ is minimized can be determined by

$\frac{\partial\langle\varepsilon\rangle}{\partial k_{1}}=0$. 
The value of $\hat{k}_{1}$ can be obtained by substituting Eq. (19) into Eq. (20). It can be shown that

$\hat{k}_{1}=\frac{\int_{0}^{T_{0.6}}\left(1-(1-T)^{3 / 2}\right) T^{n} \mathrm{~d} T}{\int_{0}^{T_{0.6}} T^{2 n} \mathrm{~d} T}$.

As can be seen in Fig. 3, $\hat{k}_{1}$ increases monotonically with the increase of $n$. This is because if both $T$ and $n$ are smaller than unity, then, to keep $k_{1} T^{n}$ constant, a larger value of $n$ needs to be used for a larger value of $k_{1}$. Substituting Eq. (21) into Eq. (19), the variation of $\langle\varepsilon\rangle$ as a function of $n$ can be evaluated. Fig. 4 shows that $\langle\varepsilon\rangle$ decreases with an increase in $n$, reaches a minimum at $n \cong 0.92$, and increases with a further increase in $n$. According to Fig. 4, the deviation of Eq. (1) from Eq. (16) is small for $n$ in the range $[0,1]$. In particular, $\langle\varepsilon\rangle$ is less than 0.01 for $n$ in the range [0.85,1]. The temporal variation of $M_{t} / M_{\infty}$ predicted by both Eqs. (1) and (16) for $n=0.92$ and $n=0.85$ are presented, respectively, in Figs. 5 and 6 . These figures reveal that for $M_{t} / M_{\infty} \leqslant 0.6$, the results based on Eq. (1) are close to those based on Eq. (16). Note that, although the optimum value of $n$ is 0.92 , the deviation arising from using Eq. (1) is acceptable $(\langle\varepsilon\rangle<0.01)$ for $n$ in the range [0.85,1]. Ritger and Peppas (1987b) pointed out that for Case II transport, the optimum values of $n$ are, respectively, $1,0.89$, and 0.85 for slabs, cylinders, and spheres. Here, we show that, instead of a single optimum value, a range of appropriate $n$ can be assumed for various devices. It should be emphasized that using Eq. (1) with $n$ in the range [0.85,1] to describe drug release for $M_{t} / M_{\infty} \leqslant 0.6$ may be acceptable for both diffusional release and Case II diffusion. This means using Eq. (1) to describe drug release cannot tell diffusional release from Case II

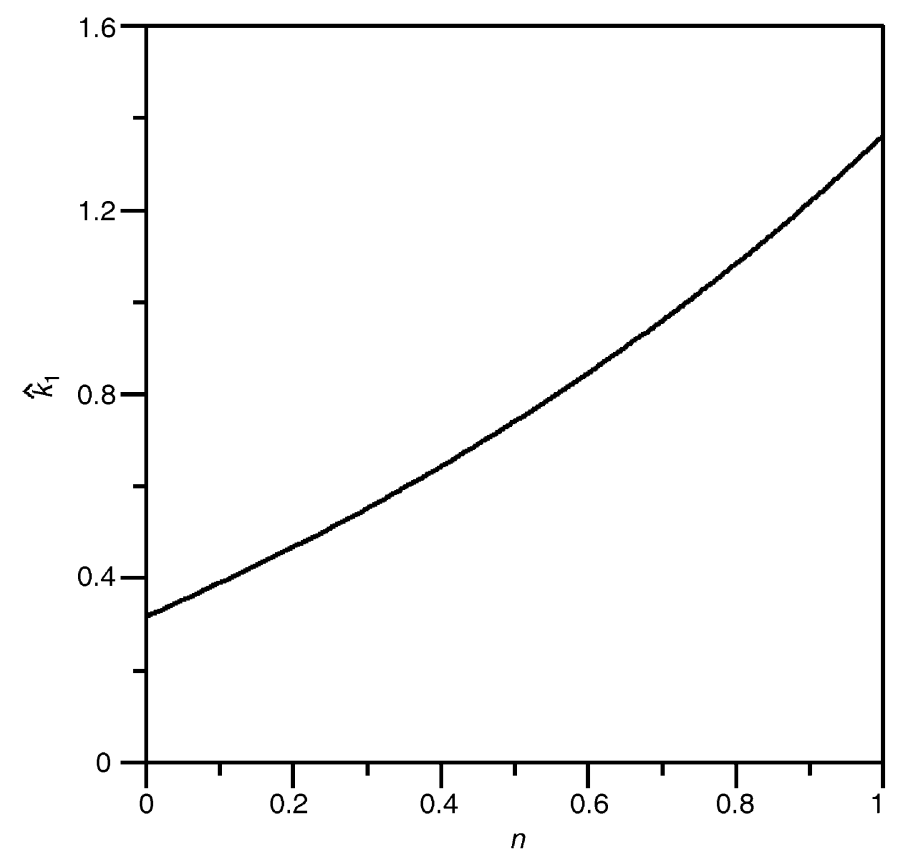

Fig. 3. Variation of $\hat{k}_{1}$ as a function of exponent $n$.

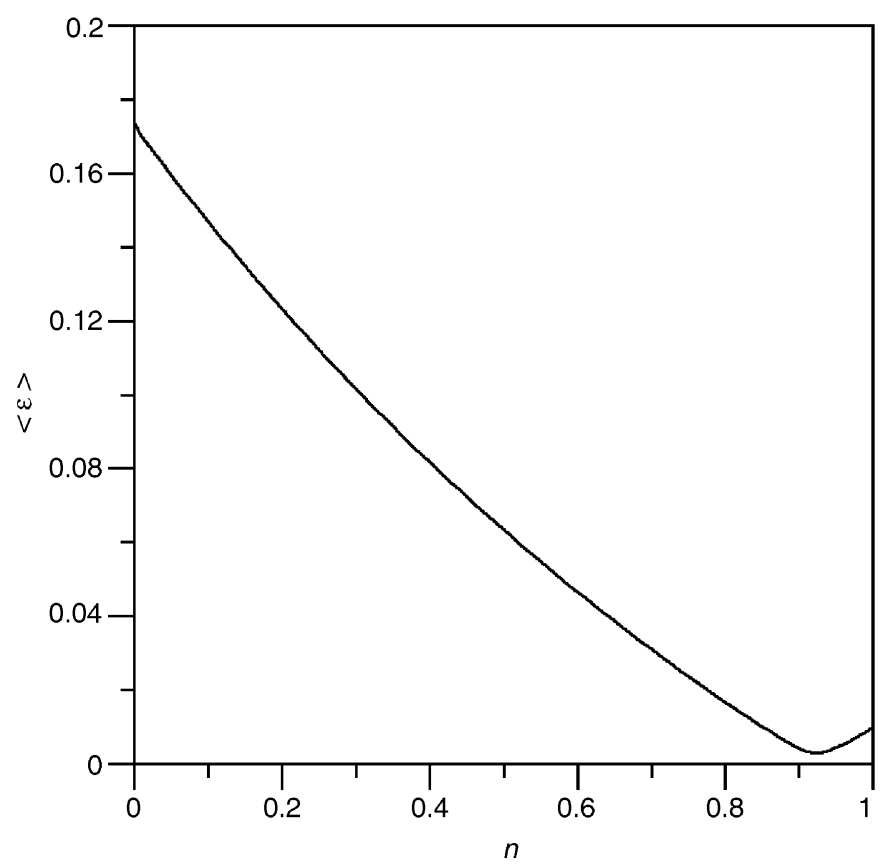

Fig. 4. Variation of root-mean-square deviation $\langle\varepsilon\rangle$ as a function of exponent $n$.

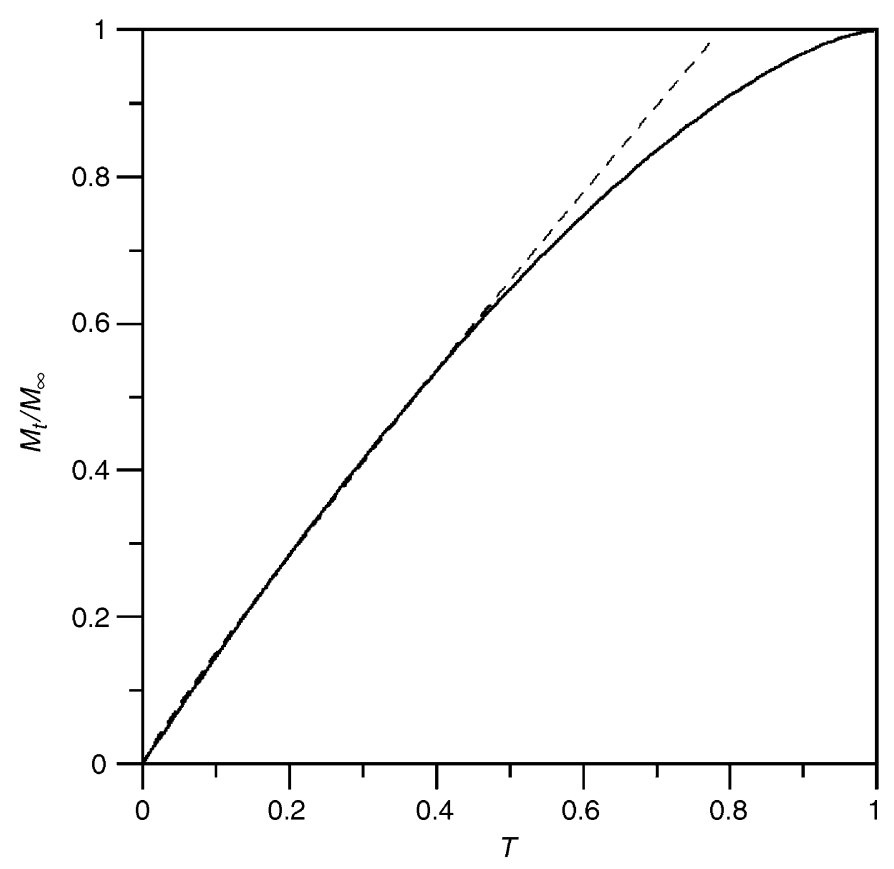

Fig. 5. Variation of cumulative release fraction $M_{t} / M_{\infty}$ as a function of scaled time T. --: Eq. (16); - - -: Eq. (1) with $n=0.92$ and $k_{1}=1.2453$.

diffusion. It may lead to inappropriate kinetic results, and the values of the parameters $n$ and $k_{1}$ thus obtained lacks of physical meaning.

In summary, the kinetics of the diffusional release for an ellipsoidal drug-delivery device is analyzed theoretically. The present analysis extends previous results in that a general geometry for drug-delivery device is considered under the 


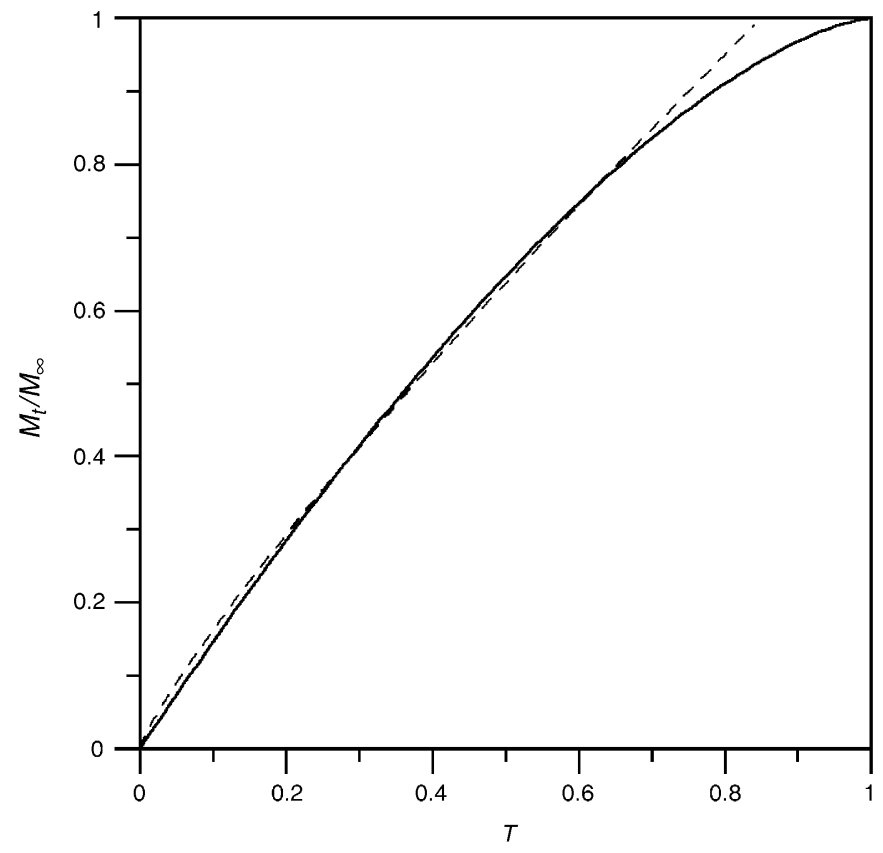

Fig. 6. Variation of cumulative release fraction $M_{t} / M_{\infty}$ as a function of the scaled time T. --: Eq. (16); - - -: Eq. (1) with $n=0.85$ and $k_{1}=1.1491$.

conditions that a moving-boundary problem needs to be solved. The result of numerical simulation reveals that, regardless of the shape of a device, the cumulative fractional release of drug can be expressed in a concise, yet general form. A comparison between the present results with a widely adopted empirical relation, Eq. (1), in the literature reveals that the latter is appropriate for the case when the cumulative fractional release of drug is below $60 \%$. However, the empirical relation may lead to inaccurate description to release kinetics.

\section{Acknowledgements}

This work is partially supported by the National Science Council of the Republic of China.

\section{References}

Abdekhodaie, M.J., 2002. Diffusional release of a solute from a spherical reservoir into a finite external volume. Journal of Pharmaceutical Sciences 91, 1803-1809.

Asrar, J., Ding, Y., Monica, R.E.L., Ness, L.C., 2004. Controlled release of tebuconazole from a polymer matrix microparticle: release kinetics and length of efficacy. Journal of Agricultural and Food Chemistry 52, 4814-4820.

Costa, P., Lobo, J.M.S., 2001. Review: modeling and comparison of dissolution profiles. European Journal of Pharmaceutical Sciences 13, 123-133.

Crank, J., 1975. The Mathematics of Diffusion. second ed. Oxford University Press, London.

Flammer, C., 1957. Spheroidal Wave Functions. Stanford University Press, Stanford.

Ham, F.S., 1959. Shape-preserving solutions of the time-dependent diffusion equation. Quarterly of Applied Mathematics 17, 137.

Higuchi, T., 1961. Rate of release of medicaments from ointment bases containing drugs in suspension. Journal of Pharmaceutical Sciences 50, 874-875.

Hopfenberg, H.B., 1976. Controlled release polymeric formulations. In: Paul, D.R., Harris, F.W. (Eds.), ACS Symposium Series 33. American Chemical Society, Washington, DC, pp. 26-31.

Lee, P.I., 1980. Diffusional release of a solute from a polymeric matrixapproximate analytical solutions. Journal Membrane Science 7, 255-275.

Liu, B.T., Hsu, J.P., 1995. Nonisothermal dissolution and crystallization of ellipsoidal particles in liquid solutions. The Journal of Chemical Physics 103, 10632-10637.

Liu, J., Xu, M., 2004. An exact solution to the moving boundary problem with fractional anomalous diffusion in drug release devices. Zeitschrift für Angewandte Mathematik und Mechanik 84, 22-28.

Narasimhan, B., Langer, R., 1997. Zero-order release of micro- and macromolecules from polymeric devices: the role of the burst effect. Journal of Controlled Release 47, 13-20.

Paul, D.R., McSpadden, S.K., 1976. Diffusional release of a solute from a polymer matrix. Journal of Controlled Release 1, 33-48.

Ritger, P.L., Peppas, N.A., 1987a. A simple equation for description of solute release: I. Fickian and non-Fickian release from non-swellable devices in the form of slabs, spheres, cylinders or discs. Journal of Controlled Release 5, 23-36.

Ritger, P.L., Peppas, N.A., 1987b. A simple equation for description of solute release: II. Fickian and anomalous release from swellable devices. Journal of Controlled Release 5, 37-42.

Zhou, Y., Chu, J.S., Wu, X.Y., 2004. Theoretical analysis of drug release into a finite medium from sphere ensembles with various size and concentration distributions. European Journal of Pharmaceutical Sciences 22, 251-259. 